\title{
Loop structure of the lowest Bloch band for a Bose-Einstein condensate
}

\author{
Dmitri Diakonov ${ }^{1,2}$, L. M. Jensen ${ }^{1}$, C. J. Pethick ${ }^{1}$, and H. Smith ${ }^{3}$ \\ 1 NORDITA, Blegdamsvej 17, DK-2100 Copenhagen Ø, Denmark \\ 2 St. Petersburg Nuclear Physics Institute, Gatchina, St. Petersburg 188300, Russia \\ 3 Ørsted Laboratory, H. C. Ørsted Institute, Universitetsparken 5, DK-2100 Copenhagen Ø, Denmark
}

(Dated: October 28, 2018)

\begin{abstract}
We investigate analytically and numerically Bloch waves for a Bose-Einstein condensate in a sinusoidal external potential. At low densities the dependence of the energy on the quasimomentum is similar to that for a single particle, but at densities greater than a critical one the lowest band becomes triple-valued near the boundary of the first Brillouin zone and develops the structure characteristic of the swallow-tail catastrophe. We comment on the experimental consequences of this behavior.
\end{abstract}

PACS numbers: 03.75.Fi, 05.30.Jp, 67.40.Db

The possibility of studying experimentally the properties of Bose-Einstein condensates in periodic potentials created by optical lattices (see e. g. [1, 2]) has stimulated a number of theoretical investigations. These include nonlinear tunneling phenomena 3, 4, Bloch oscillations [5, 6], stability of Bloch waves [7], and oscillations of condensates and localized excitations [8]. In a two-level model of nonlinear Landau-Zener tunneling, evidence was found for a loop structure in the adiabatic energy levels [3]. Recently Bronski et al. [9] discovered a remarkable exact solution of the Gross-Pitaevskii equation for a condensate in a one-dimensional optical lattice for a quasimomentum corresponding to the boundary of the first Brillouin zone. The reason for an exact solution being possible is that the spatially dependent parts of the lattice potential and the internal field due to interparticle interactions cancel. Subsequently, in Ref. 10] it was shown by numerical studies that the current of the condensate for quasimomenta in the vicinity of the zone boundary had a singular behavior, and it was speculated that the chemical potential of the condensate became multivalued near the zone boundary. In this Letter we use analytical and numerical methods to show that the lowest band has the structure associated with the swallow-tail catastrophe [11], and we comment on implications of this result for experiment.

The potential $V(x)$ produced by a one-dimensional standing light wave has the form

$$
V(x)=V_{0} \sin ^{2} \frac{\pi x}{d},
$$

where $d$ is the period of the lattice. We shall assume that the condensate may be described by the Gross-Pitaevskii functional for the energy density, which when averaged over a lattice period is given by

$$
\mathcal{E}=\frac{1}{d} \int_{-d / 2}^{d / 2} d x\left[\frac{\hbar^{2}}{2 m}\left|\frac{d \psi}{d x}\right|^{2}+V(x)|\psi|^{2}+\frac{U_{0}}{2}|\psi|^{4}\right],
$$

and the mean number density $n$ is given by

$$
n=\frac{1}{d} \int_{-d / 2}^{d / 2} d x|\psi|^{2} .
$$

Here $\psi$ is the wave function of the condensate, $m$ is the particle mass, and the effective two-body interaction is given by $U_{0}=4 \pi \hbar^{2} a / m$, where $a$ is the scattering length for two-body collisions. The condensate wave function satisfies the nonlinear Schrödinger equation

$$
-\frac{\hbar^{2}}{2 m} \frac{d^{2} \psi}{d x^{2}}+\left[V(x)+U_{0}|\psi|^{2}-\mu\right] \psi=0 .
$$

The quantity $\mu$ is the chemical potential, and this depends both on the mean density of particles, and on the quasimomentum. We shall look for solutions where the mean density is independent of position, and therefore these must satisfy the condition for quasi-periodicity,

$$
\psi(x+d)=\exp (i k d) \psi(x)
$$

for all $x$. The quantity $\hbar k$ is the quasimomentum of the condensate. Our objective is to find the energy per particle, the chemical potential, and the current density of nonlinear Bloch waves as a function of $k$ and of $n$. The current density of the condensate is given by

$$
j(k)=\frac{\hbar}{2 m i}\left(\psi^{*} \frac{d \psi}{d x}-\psi \frac{d \psi^{*}}{d x}\right) .
$$

It is independent of $x$ provided $\psi$ satisfies Eq. (4). By direct calculation one can demonstrate that

$$
j(k)=\frac{1}{\hbar} \frac{\partial \mathcal{E}(k, n)}{\partial k} .
$$

The quantity $j / n$ is the mean particle velocity.

Without loss of generality, we may choose $\psi(0)$ to be real. Since the periodic potential is even in $x$, one infers from the quasi-periodicity condition (5) that the real part of the condensate wave function is an even function of $x$ and the imaginary part an odd function.

The solution at the zone center $(k=0)$ corresponds to a purely real and periodic wave function, $\psi(x+d)=\psi(x)$. Also at the zone boundary $(k= \pm \pi / d)$ there is a purely real solution which is antiperiodic, $\psi(x+d)=-\psi(x)$. Since $\psi$ is real, these solutions have zero current, $j=$ 0 . Other solutions are complex and the current is nonzero. Since the potential in Eq. (14) is real, it follows that 
$\psi_{k}(x)=\psi_{-k}^{*}(x)$. Naturally, all physical quantities are periodic in $k$ with period $2 \pi / d$.

The structure of the energy as a function of $k$ near the zone boundary may be brought out by making a variational calculation of the energy. We use a trial function of the form

$$
\psi(x)=\sqrt{n}\{\cos \alpha \exp i k x+\sin \alpha \exp (i[k-2 \pi / d] x)\},
$$

where $\alpha$ is a variational parameter. This is similar to that used in the almost-free-electron approximation for the band structure of a single particle, and it also corresponds to the two-level model studied in Ref. [3]. When the trial function (8) is inserted into the energy functional (2), it becomes

$$
\begin{aligned}
\mathcal{E}=n(\bar{\epsilon} & \left.+\frac{\Delta \epsilon}{2} \cos 2 \alpha\right)+\frac{n V_{0}}{2}\left(1-\frac{1}{2} \sin 2 \alpha\right) \\
& +\frac{n^{2} U_{0}}{2}\left(1+\frac{1}{2} \sin ^{2} 2 \alpha\right),
\end{aligned}
$$

where the three terms are the kinetic, potential and interaction energies, respectively, with $\bar{\epsilon}=\left(\epsilon_{k}+\epsilon_{k-2 \pi / d}\right) / 2$, where $\epsilon_{k}=\hbar^{2} k^{2} / 2 m$, and $\Delta \epsilon=\epsilon_{k}-\epsilon_{k-2 \pi / d}=$ $\left(2 \pi \hbar^{2} / m d\right)(k-\pi / d)$. The optimal value of $\alpha$ is obtained by requiring (9) to be stationary with respect to variations in $\alpha$, and the chemical potential $\mu$ is then found by differentiating the resulting energy with respect to the density, $\mu=\partial \mathcal{E} / \partial n$.

At the zone boundary $k= \pm \pi / d$ the stationarity of (9) yields two solutions: $\sin 2 \alpha=V_{0} / 2 n U_{0}$ and $\cos 2 \alpha=0$. The first solution, which requires that the density exceed a critical value $n_{\mathrm{c}}$ given by

$$
n_{\mathrm{c}}=V_{0} / 2 U_{0},
$$

has an energy density

$$
\mathcal{E}_{1}=n\left(\frac{\hbar^{2} \pi^{2}}{2 m d^{2}}+\frac{V_{0}}{2}\right)+\frac{n^{2} U_{0}}{2}-\frac{V_{0}^{2}}{16 U_{0}},
$$

a chemical potential

$$
\mu_{1}=\mu_{\mathrm{c}}+\left(n-n_{\mathrm{c}}\right) U_{0},
$$

where

$$
\mu_{\mathrm{c}}=V_{0}+\frac{\hbar^{2} \pi^{2}}{2 m d^{2}},
$$

and a current density

$$
j= \pm \frac{\hbar \pi}{m d} \sqrt{n^{2}-n_{\mathrm{c}}^{2}} .
$$

The two possible signs for the current density reflect the fact that if $\alpha$ is a solution to the stationarity condition, so is $\pi / 2-\alpha$.

Equations (10)-(14) are in fact exact, the variational trial function (8) being in this case identical with the analytical solution found in Refs. 9, 10]. At the critical density when the analytical solution first appears with $\alpha=\pi / 4$, the current is zero since $\psi(x)$ is real. We note that the critical chemical potential is the height of the barrier, plus the kinetic energy of a free particle with a momentum equal to the quasimomentum at the zone boundary.

For $n>n_{\mathrm{c}}$ the exact solution (8) with $\sin 2 \alpha=$ $V_{0} / 2 n U_{0}<1$ is complex and carries a non-zero current. Remarkably, although this state has $k= \pm \pi / d$, it has neither the highest energy nor the highest chemical potential of all states in the band: the real antiperiodic wave function, also with $k= \pm \pi / d$, has higher $\mu$, and it corresponds to the second solution of the stationarity condition, $\cos 2 \alpha=0$ or $\alpha=\pi / 4$ (the other possibility, $\alpha=3 \pi / 4$, belongs to the next, excited Bloch band and will not be considered here). This solution exists at all densities and corresponds to a chemical potential $\mu_{2}=\mu_{\mathrm{c}}+3\left(n-n_{\mathrm{c}}\right) U_{0} / 2$ which is higher than $\mu_{1}$ at $n>n_{\mathrm{c}}$.

For a general value of $k$, the $\Delta \epsilon$ term in Eq. (9) for the total energy tends to make the roots of the stationarity condition initially at $\alpha=\pi / 4$ and $\alpha=$ $\left[\arcsin \left(V_{0} / 2 n U_{0}\right)\right] / 2$ approach one another, and the two roots merge and disappear for a sufficiently large value of the magnitude of $\Delta \epsilon$. The energy per particle obtained using the trial function (8) is shown in Fig. 1, where one sees the swallow-tail structure near the zone boundary. Throughout, we shall measure energies in units of $\hbar \omega$, where $\omega=\left(2 V_{0} / m\right)^{1 / 2} \pi / d$ is the angular frequency of small oscillations about a minimum of the potential.

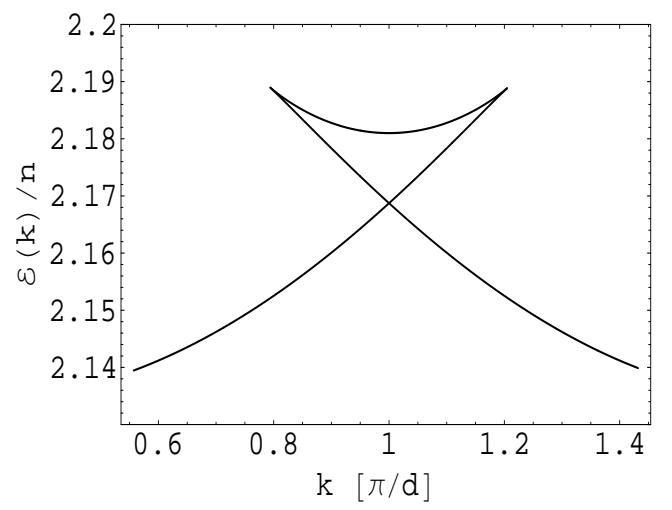

FIG. 1: Swallow-tail structure of the energy per particle as a function of $k$ for $V_{0}=3 \hbar \omega$ and $n / n_{\mathrm{c}}=1.20$.

A quantitative analysis shows that the trial form (8) is a good approximation near the zone boundary provided $\hbar^{2} \pi^{2} / 2 m d^{2} \gg n U_{0}$. The analytical calculations may be improved by including plane-wave components with wave numbers that differ from the ones in Eq. (8) by multiples of the reciprocal lattice vector $2 \pi / d$.

We now demonstrate that the swallow-tail structure also arises in numerical solutions of the full GrossPitaevskii equation (位. At condensate densities below 
the critical one, the wave function and dispersion relation for nonlinear Bloch waves are qualitatively similar to those for a single particle. To illustrate this, we again consider the case where the potential barrier is $V_{0}=3 \hbar \omega$.

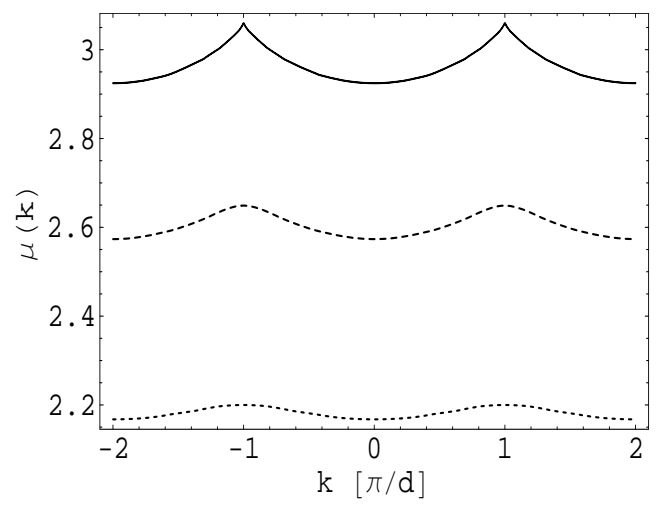

FIG. 2: Chemical potential $\mu$ in units of $\hbar \omega$ as a function of quasimomentum for densities $n / n_{\mathrm{c}}=0.57$ (dotted), 0.78 (dashed), and 0.99 (full line).

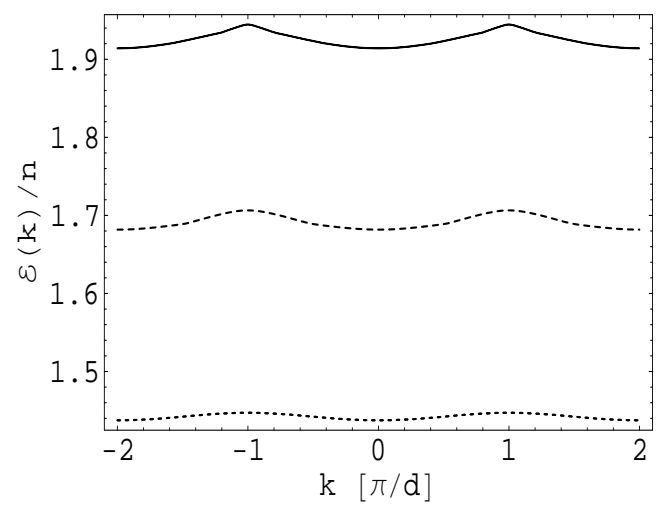

FIG. 3: Energy per particle, $\mathcal{E} / n$, in units of $\hbar \omega$ as a function of quasimomentum for densities $n / n_{\mathrm{c}}=0.57$ (dotted), 0.78 (dashed), and 0.99 (full line).

In Figs. 2 and 3 we plot the chemical potential $\mu(k, n)$ and the energy per particle for several values of density $n<n_{\mathrm{c}}$. In all cases the chemical potential lies below the top of the potential barriers. Therefore, the energy bands are relatively narrow as the motion of the condensate involves tunneling through the barriers. The difference between the chemical potential and the energy per particle reflects the nonlinear character of the interparticle interaction.

In Fig. 4 we show the current density $j(k, n)$ of the nonlinear waves for the same number densities as in Figs. 2 and 3 . The current goes to zero both at the zone center and the zone boundary. At the critical density the real antiperiodic wave function corresponding to the top of the band and having $k= \pm \pi / d$ coincides with the

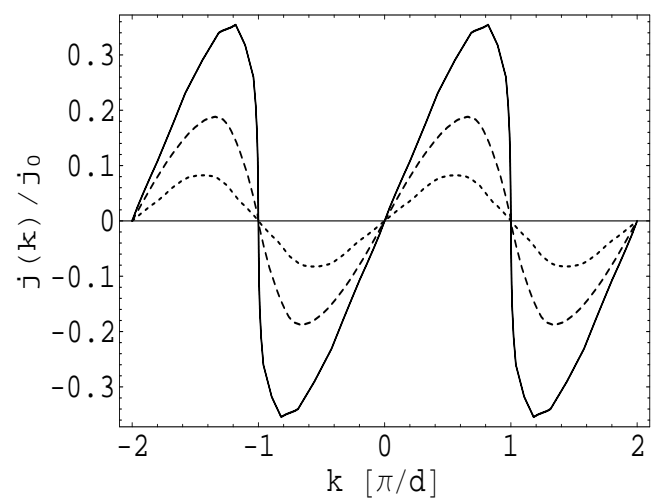

FIG. 4: The current density $j$ as a function of quasimomentum at densities $n / n_{\mathrm{c}}=0.57$ (dotted), 0.78 (dashed), and 0.99 (full line). Here $j_{0}=\pi \hbar n / m d$ is the current density at $k=\pi / d$ in the absence of a lattice.

analytical solution,

$$
\psi_{\mathrm{c}}^{k= \pm \pi / d}(x)=\sqrt{\frac{V_{0}}{U_{0}}} \cos \frac{\pi x}{d} .
$$

The current density $j\left(k, n_{\mathrm{c}}\right)$ goes to zero at $k= \pm \pi / d$ but has an infinite derivative at this point, see Fig. 4. Consequently, $\mu\left(k, n_{\mathrm{c}}\right)$ develops a singularity at $k= \pm \pi / d$ (see Fig. 2), as does the energy density.

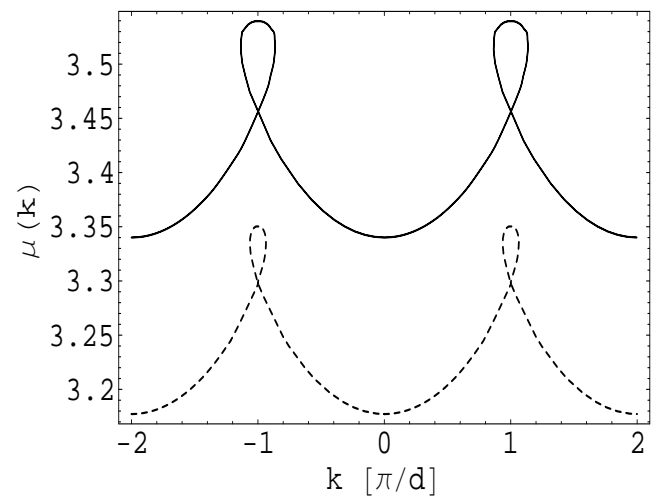

FIG. 5: Chemical potential $\mu$ as a function of quasimomentum for densities $n / n_{\mathrm{c}}=1.14$ (dashed) and 1.25 (full line).

For $n>n_{\mathrm{c}}$ the Bloch wave evolves from a real periodic wave function at $k=0$ to the analytical plane-wave solution (8) at $k=\pi / d$, which, however, is now complex. At $k=\pi / d$ there is a second solution equal to the complex conjugate of the first one and, in addition, a third one which is real, and which therefore carries no current. Also for $k$ close to $\pi / d$ one finds three solutions for a given $k$. We plot the chemical potential $\mu(k, n)$, the current density $j(k, n)$, and the energy per particle $\mathcal{E}(k, n) / n$ in Figs. $5-7$, respectively, for two values of the density $n>n_{\mathrm{c}}$. We note that $j(k, n)$ calculated from the 
wave function using Eq. (6) is consistent with the relation (7).

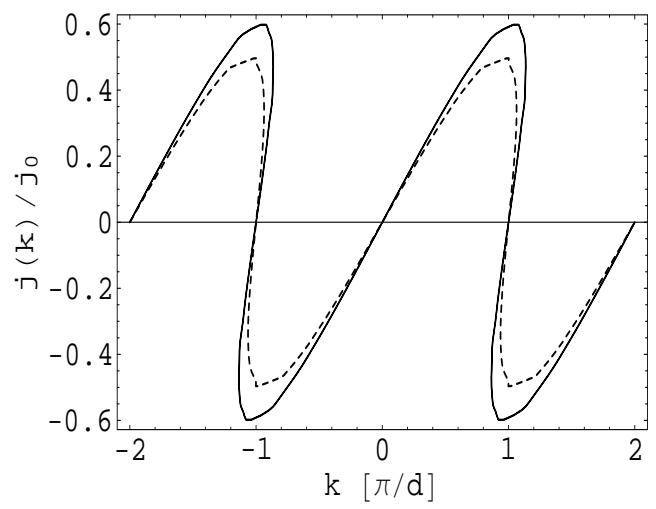

FIG. 6: Current density $j$ as a function of quasimomentum for densities, $n / n_{\mathrm{c}}=1.14$ (dashed) and 1.25 (full line). The unit of current $j_{0}$ is the same as in Fig. 4.

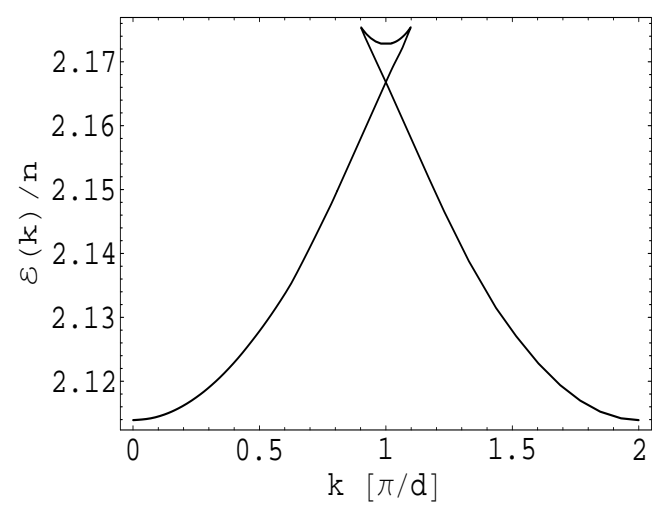

FIG. 7: Energy per particle $\mathcal{E} / n$ of a Bloch wave as a function of quasimomentum for a condensate density $n / n_{\mathrm{c}}=1.20$.

The unusual structure of the energy bands has important implications for experiment. If a single particle in a periodic potential is subjected to a small constant force, $k$ varies linearly in time, and the particle velocity and position execute Bloch oscillations. Such behavior has been observed in optical lattices for thermal atoms above the Bose-Einstein transition temperature 12]. Similar arguments apply to the motion of a Bose-Einstein condensate, provided the energy of the condensate in the lowest band is a continuous, single-valued function of $k$. Bloch oscillations have indeed been observed for condensates [2]. At densities greater than $n_{\mathrm{c}}$, the situation is different because of the swallow-tail structure of the energy band. When a weak force is applied to a condensate initially at rest, in the simple picture of the motion $k$ will increase and with time will reach the value $\pi / d$. With further increase of $k$, the system will continue on the branch of the spectrum for $k>\pi / d$ which has the same slope at $k=\pi / d$ as that of the initial branch. With this choice of branch, the wave function varies continuously as $k$ increases past $\pi / d$, whereas there is a discontinuous change in the wave function of the lowest energy state. A dramatic manifestation of this is that the current density in the lowest energy state changes sign at $k=\pi / d$. With time, $k$ will eventually reach the tip of the swallow tail, and it then becomes impossible to describe the condensate motion in terms of the usual adiabatic picture, as was found in Ref. [3] for a two-level model. How to treat the dynamics under such conditions remains a challenging open problem.

The experiments on Bloch oscillations in Ref. [2] were carried out in a regime in which the interaction energy was small compared with the depth of the potential due to the optical lattice, and therefore one would not have expected the energy band to have the swallow-tail structure. It is important to extend such experiments into the regime in which the strong nonlinear effects predicted here should occur. Theoretically, the stability of a condensate moving in a periodic potential has been examined for relatively low values of the two-body interaction [7, and such studies should be extended to higher values of the interaction, where the swallow tail develops.

We are grateful to D. Aristov and S. J. Chang for very useful discussions.
[1] B. P. Anderson and M. A. Kasevich, Science 282, 1686 (1998); S. Burger, F. S. Cataliotti, C. Fort, F. Minardi, A. Trombettoni, A. Smerzi, and M. Inguscio, Phys. Rev. Lett. 86, 4447 (2001); F. S. Cataliotti, S. Burger, C. Fort, P. Maddaloni, F. Minardi, M. Inguscio, M. L. Chiofalo, and M. P. Tosi, Science 293, 843 (2001).

[2] O. Morsch, J. H. Müller, M. Cristiani, D. Ciampini, and E. Arimondo, Phys. Rev. Lett. 87, 140402 (2001).

[3] B. Wu and Q. Niu, Phys. Rev. A 61, 023402 (2000).

[4] O. Zobay and B. M. Garraway, Phys. Rev. A 61, 033603 (2000).

[5] K. Berg-Sørensen and K. Mølmer, Phys. Rev. A 58, 1480 (1998).
[6] D.-I. Choi and Q. Niu, Phys. Rev. Lett. 82, 2022 (1999).

[7] B. Wu and Q. Niu, cond-mat/0009455.

[8] A. Trombettoni and A. Smerzi, Phys. Rev. Lett. 86, 2353 (2001).

[9] J. C. Bronski, L. D. Carr, B. Deconinck, and J. N. Kutz, Phys. Rev. Lett. 86, 1402 (2001); J. C. Bronski, L. D. Carr, B. Deconinck, J. N. Kutz, and K. Promislow, Phys. Rev. E 63, 036612 (2001).

[10] B. Wu, R. B. Diener, and Q. Niu, cond-mat/0109183.

[11] R. Thom, Structural Stability and Morphogenesis (Benjamin, Reading, Massachusetts, 1975) p. 64.

[12] M. Ben Dahan, E. Peik, J. Reichel, Y. Castin, and C. Salomon, Phys. Rev. A 76, 4508 (1996). 